\title{
Liver Cancer Patient Classification on a Multiple-Stage using Hybrid Classification Methods
}

\author{
Orasa PATSADU*, Pongsakorn TANGCHITWILAIKUN and \\ Supanut LOWSUWANKUL
}

\author{
Faculty of Science and Technology, Rajamangala University of Technology Krungthep, \\ Bangkok 10120, Thailand
}

("Corresponding author's e-mail: orasa.p@mail.rmutk.ac.th)

Received: 21 November 2019, Revised: 15 October 2020, Accepted: 20 November 2020

\begin{abstract}
This paper proposes a model to detect liver cancer patients and estimate the abnormality level of livers using a classification method based on an Indian liver patient dataset. The dataset is prepared by 3 processes: preliminary study, data cleansing, and handling imbalanced class to build the model based on multiple-stages using hybrid classification methods. The $1^{\text {st }}$ stage is liver cancer patient detection. The $2^{\text {nd }}$ stage is abnormality level of liver estimation, as divided using the DeRitis Ratio. The abnormality level of livers is divided into 3 levels: low, medium, and high, called ALL framework. Machine learning method is used to build multiple classification stages, which consist of Multilayer Perceptron, Logistic Regression, and Random Forest. The experimental results demonstrate that the $1^{\text {st }}$ model (stage I) can detect liver cancer patient with $78.88 \%$ accuracy. The $2^{\text {nd }}$ model (stage II) achieves accuracy of $99.83 \%$ for abnormality level of liver estimation. In addition, we compare our proposed model with another dataset. Our proposed model also outperforms detection with 76.73 and $98.26 \%$ accuracy in stage I and stage II, respectively. Our proposed model is a benefit for physicians to support diagnosis and treatment, especially in the case of physicians desiring an intelligent decision support system.
\end{abstract}

Keywords: Liver cancer, Hybrid classification methods, Abnormality level of liver estimation, Multiplestage classifier, Decision support system

\section{Introduction}

The most prevalent type of cancer is liver cancer [1]. "The symptoms of liver cancer are difficult to be discovered in the initial phase. If it can be discovered in an early stage and patient immediately obtains treatment, it will increase patient's survival rate" [2-4]. In Thailand, liver cancer is the main cause of mortality; especially, men (aged above 40 years old) have a risk of liver cancer disease that is higher than women [5]. Tumors of liver cancer are divided into 2 phases: the initial phase and the severe phase [3]. This paper aims to classify Hepatocellular Carcinoma (HCC) [6], which is an important cause of death of liver patients in Thailand and Southeast Asia [7]. "The factors that cause HCC are cirrhosis, alcohol drinking, Hepatitis B, or receiving drugs or chemicals for a long time" $[1,3,8]$.

There have been numerous researches which have used Alpha-fetoprotein (AFP) to detect liver cancer $[7,9,10]$. AFP is a tumor marker. Results indicate that approximately $50 \%$ of HCC patients have high AFP values (AFP > 200). However, AFP value may only be checked every 6 months, which makes it difficult to detect symptoms at an early stage [9]. There has been a research study on analyzing appropriate AFP values for Hepatocellular Carcinoma detection. Nevertheless, this method is still not able to detect liver cancer at an early stage [7,10]. Moreover, there have been numerous researches which have used hybrid classification methods to detect HCC at an early stage $[11,12]$. Nevertheless, previous 
researches have rarely defined methods to estimate abnormality levels of livers so as to be used for diagnosis and treatment. Most of the research has focused on other information, such as type of disease, stage of disease, size, number, and progression [13,14]. As well as these factors, treatment method selection also depends on liver function. Therefore, we should have a diagnosis support system to detect liver cancer patients at an early stage $[15,16]$ that could promptly estimate abnormality levels of livers. This is important to physicians, especially in the case of physicians desiring an intelligent system to support diagnosis and treatment. This has become our motivation.

Therefore, this paper proposes a multiple-stage classification method. The $1^{\text {st }}$ stage is liver cancer patient detection. The $2^{\text {nd }}$ stage is abnormality level of liver estimation as an obtained result from the $1^{\text {st }}$ stage. A Liver Patient Dataset (shown in Table 1) is prepared for model building. The classification methods used to build models were Multilayer Perceptron (MLP), Logistic Regression, and Random Forest. In addition, our proposed model uses hybrid classification methods based on Voting, with an average of probabilities of each model to select the best answer.

The remaining structure of this paper consists of related work, materials and methods, experiment result and demonstration, and conclusions and future work, as described in the next section.

\section{Related work}

The literature review is divided into 2 issues: liver cancer patient detection and abnormality level of liver estimation.

\section{Model of liver cancer patient detection}

There have been numerous researches which have proposed models to detect liver cancer disease using data mining techniques based on UCI repository datasets [2]. Kumar and Thakur [17] proposed a liver cancer detection method using Variable NWFKNN. There were 3 datasets, BUPA, ILPD, and MPRLPD. The results showed that this method can detect liver cancer disease with higher accuracy than NWKNN.

Baitharua and Pani [18] proposed a method to detect liver cancer disease which used several classifiers "such as DT, NB, ANN, ZeroR, 1BK, and VFI" [18]. The results showed that ANN could detect liver cancer disease with an accuracy of $71.59 \%$ when compared to other classifications.

Sontakke et al. [4] proposed 2 methods to detect liver cancer disease based on an Indian liver patient dataset and gene expression. The classification methods used to build models were SVM and BPNN. This paper handled missing values and performed oversampling and undersampling. The results showed that BPNN could detect liver cancer disease with higher accuracy than SVM.

Ma'aitah et al. [19] proposed a method to detect alcoholic drinks in the body using a Fuzzy Neural system based on a BUPA dataset. This paper performed data preprocessing using data normalization. Then, fuzzy logic was used to detect liver cancer disease. The results showed that the system could detect liver cancer disease with an accuracy of $97.39 \%$ (5 feature selection of $\left.\mathrm{x}_{6} \geq 3\right)$ and an accuracy of $72 \%$ ( 5 feature selection of $\mathrm{x}_{6}>5$ ).

In addition, Pasha and Fatima [1] proposed a method to detect liver cancer disease. Adaboost, Logit boost, Bagging, and Grading algorithms were used to detect liver cancer disease based on an Indian liver patient dataset. This paper focused on 3 main points of a model, accuracy, error, and processing time. The results showed that Grading had high performance, with an accuracy of $71.3551 \%$, less error, and less processing time when compared to other techniques.

Kumar and Sahu [20] presented a method to detect liver cancer disease based on a liver dataset from UCI ( 8 features and 768 instances, 1 class). C4.5, RF, Cart, Random tree, and REP tree were used to detect liver cancer disease. The results showed that RF (6 features using $80-20 \%$ ) could detect liver cancer disease with an accuracy of $79.22 \%$.

Aiswarya et al. [21] proposed liver cancer disease detection which compared 2 classifiers, RF and NB, based on an Indian liver patient dataset. The results showed that RF had higher accuracy than NB.

Alaybeyoglu and Mulayim [15] proposed an expert system to detect liver cancer disease and breast cancer using firefly algorithm based on an Indian liver patient dataset and a Wisconsin dataset. The 
results showed that the system could detect liver cancer disease with an accuracy of $92 \%$ and could detect breast cancer with an accuracy of $94.81 \%$.

Priya et al. [22] presented a method to detect liver cancer disease using a classification method based on an Indian liver patient dataset. There were 4 phases: min-max normalization, PSO feature selection, classification method (J48, SVM, MLP, Bayesian network, RF), and evaluate model using MAE, RMSE, and accuracy. The results showed that J48 could detect liver cancer disease with an accuracy of $95.04 \%$ using PSO feature. Lastly, this model could be applied to detect heart disease. Pathan et al. [23] also proposed liver cancer disease detection using a dataset which resembled that of Priya et al. [22]. Data were prepared using K-Means to select attributes. Classification methods used to detect liver cancer disease were NB, Adaboost, J48, Bagging, and RF. The results showed that RF could detect liver cancer disease with high accuracy.

Jacob et al. [16] presented a system to detect liver cancer disease based on an Indian liver patient dataset for diagnosis support for physicians. There were 4 classifiers for liver cancer disease detection, LR, SVM, KNN, and ANN. The results showed that ANN achieved high accuracy of $98 \%$. Therefore, ANN was used to develop a system.

Muthuselvan et al. [24] proposed a method to detect liver cancer disease with 4 classifiers, NB, J48, Random Tree, and K-star, based on an Indian liver patient dataset. Data preprocessing used data cretization, data normalization, and resampling. The results showed that Random Tree achieved high accuracy of $74.2 \%$. However, it used more processing time than other classifiers.

Furthermore, there have been several researches which proposed a liver cancer detection method using CT scan imaging. Rajesh and Priyadharson [25] presented liver cancer disease detection using ultrasound imaging. Ultrasound imaging was used to extract features for model building using penguin search optimization algorithm. Neural Network was used to build a model for liver cancer disease detection. The results showed that this method could detect liver cancer disease with an accuracy of 98.92 $\%$.

Das et al. [26] proposed liver cancer disease detection using CT scan imaging. Spatial fuzzy clustering approach was used to extract features for model building. MLP and C4.5 were used to compare performance. The results showed that this method could detect liver cancer disease using MLP and C4.5 with accuracies of 89.15 and $95.02 \%$, respectively.

Chen et al. [27] proposed liver cancer disease detection using histopathology $\mathrm{H}$ and $\mathrm{E}$ imaging which used deep learning (convolutional neural network) to build a model. The results showed that this method could detect liver cancer disease with an accuracy of $96.0 \%$. In addition, this method could detect gene mutations in liver cancer with AUCs of 0.71 - 0.89. Also, Almotairi et al. [28] presented liver cancer detection using deep learning (convolutional neural network) to build a model which use CT scan imaging. From the experiment, the results showed that this method could detect with an accuracy of 99.90 $\%$.

Moreover, to enhance the performance of detection using individual classifiers, there have been several researches that used hybrid classification methods. Kaur et al. [11] proposed liver cancer disease detection using hybrid classification methods (SVM, RF, Naïve Bayes, SMO, and J48). The results showed that this method could detect liver cancer disease with accuracy.

Nahar et al. [29] presented liver cancer detection using an Indian liver patient dataset which used hybrid classification methods by comparing several techniques "such as AdaBoost, LogitBoost, BeggRep, BeggJ48, and Random Forest to select the best answer" [29]. The results showed that LogitBoost had high accuracy of $71.53 \%$ when compared to other techniques.

Sathurthi and Saruladha [30] proposed liver cancer disease detection using patient information which used hybrid classification methods such as J48, C4.5, and Naïve Bayes. They compared 2 algorithms to select the best answer, adaboost algorithms and decision stump. The results showed that this method could detect liver cancer disease with accuracy.

Aswathy [31] proposed a hybrid classification method to detect liver cancer disease based on an Indian liver patient dataset. RF, Adaboost, DT, MNB, GNB, KNN, SGD, SVC, and oneVsRest were used to detect liver cancer disease. Data preprocessing used normalization, assigning quantiles ranges, oversampling (SMOTE), undersampling, number of samples reducing, binary encoding, and 1 hot 
encoding. The results showed that not only did data preprocessing improve the accuracy of liver cancer disease detection, but also imbalancing class using SMOTE was increased in performance of liver cancer disease detection using RF and Adaboost classifiers.

Panthong and Srivihok [12] presented hybrid classification methods to detect liver cancer disease. This paper used a dataset from a cancer hospital in Thailand. Features were extracted from the dataset and the model was built using NB, DT, and KNN. In addition, there were 2 hybrid classification methods (Bagging and Adaboost) used to enhance the accuracy of the model. The results showed that the system could detect liver cancer disease with an accuracy of $78.36 \%$ (LC_dataset I) and an accuracy of $84.82 \%$ (LC_dataset II).

Rajathi and Jiji [32] proposed a hybrid classification method to detect liver cancer disease using CT scan imaging. Then, features were extracted using image processing and selection of features done using WOA-SA. Classification methods used to build a model were SVM, KNN, and RF. The results showed that the system could detect liver cancer disease with $98 \%$ accuracy and performance compared with 2 algorithms had a $95 \%$ confidence interval.

In addition, the causes of low accuracy were imbalancing and insufficient data to find a pattern. Moreover, some data may not have been randomized before model building, which may have caused underfitting. Santos et al. [33] "presented a technique to solve problems about unbalanced data." This study used K-Means method to select representative data. Logistic Regression and Neural Network were used to evaluate the model. The results showed that Neural Network achieved higher performance than Logistic Regression.

\section{Model of abnormality level of liver estimation}

A method of abnormality level of liver estimation has rarely been proposed in order to divide abnormality levels of liver which may occur in liver cancer patient. Abnormality level of liver information is used to support diagnosis and to plan further treatment by physicians. There have been several researches which have proposed methods to detect liver substance severity level, type of disease, phase of disease, size, number, and progression. Singh and Pander [34] proposed a system to detect liver severity level. The severity level was divided into 4 levels, A, B, C, and D. A was a low severity level. D was a very high severity level. "Linear discriminant analysis, diagonal linear discriminant analysis, quadratic discriminant analysis, diagonal quadratic discriminant analysis, classification and regression tree, and k-nearest neighbors were used to build model" [34]. The results showed that k-nearest neighbors could detect liver severity level with an accuracy of $92.53 \%$.

Vijayarani and Dhayanand [13] presented a method to detect liver cancer disease using NB and SVM based on an Indian liver patient dataset. "After that, this method detects the type of liver cancer disease (cirrhosis, bile duct, chronic hepatitis, liver cancer, and acute hepatitis)" [13]. The results showed that SVM could detect liver cancer disease with high accuracy when compared to NB.

Rouhani and Haghighi [14] presented a system to detect liver cancer disease based on classification method. SVM and ANN were used to detect liver cancer disease. "In addition, this paper classifies type of disease and phase of disease using RBF, GRNN, PNN, LVQ, and SVM based on 6 classes such as hepatitis B (2 stages), hepatitis C (2 stages), non-viral hepatitis, and no-hepatitis" [14]. The results showed that the system could detect liver cancer disease with an approximate accuracy of $97 \%$.

\section{Materials and methods}

In this section, we explain the process of liver cancer patient detection and estimating abnormality levels of liver. The method is divided into 3 parts: data collection, data preprocessing, and liver cancer patient detection based on a multiple-stage, as shown in Figure 1. 
http://wjst.wu.ac.th

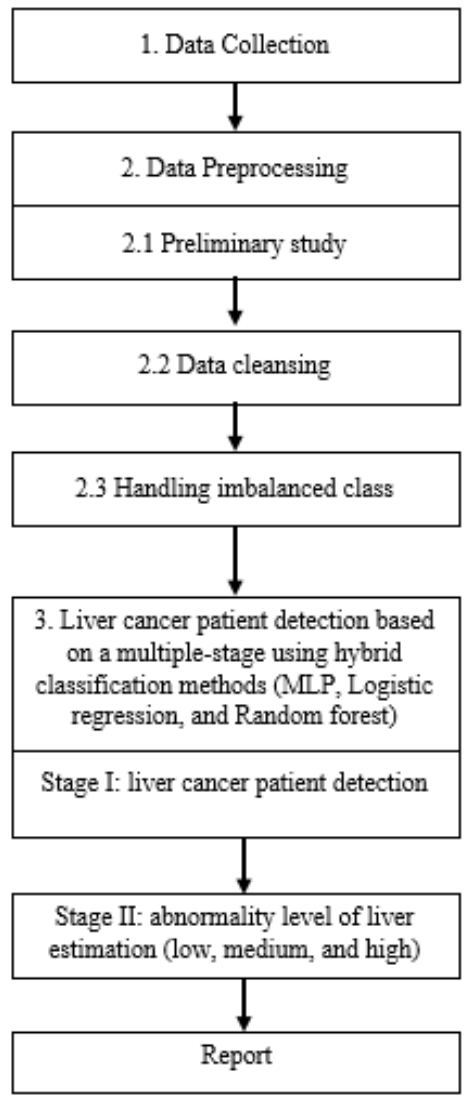

Figure 1 Overview of proposed system.

From Figure 1, each part of our proposed model is explained in the next stage.

\section{Data collection}

"We use a dataset from UCI repository based on an Indian liver patient dataset (shown in Table 1) by collecting from Andhra Pradesh in India" [35]. The dataset consisted of 583 sample subjects (416 subjects for Hepatocellular Carcinoma, and 167 subjects for normal subjects).

Table 1 Characteristics and descriptions [35].

\begin{tabular}{ll}
\hline \multicolumn{1}{c}{ Feature name } & \multicolumn{1}{c}{ Description } \\
\hline Age & Patient age \\
Gender & Patient gender \\
Total Bilirubin & Blood test evaluates amount of a substance \\
Direct Bilirubin & Bilirubin in a blood or urine \\
ALP & Blood test evaluates amount of enzyme in patient blood \\
SGPT & Blood test evaluates level of alanine aminotransferase \\
SGOT & Blood test evaluates level of aspartate aminotransferase \\
Total Proteins & A total protein test evaluates amount of protein in patient blood \\
Albumin & Blood test evaluates amount of albumin in patient blood \\
A/G ratio & Blood test evaluates level of protein in patient body \\
Class & Normal subject (No) or Hepatocellular Carcinoma patient (Yes) \\
\hline
\end{tabular}




\section{Data preprocessing}

To enhance effective and accuracy of detection, data should be prepared as follow as:

\section{Preliminary study}

We tested the independence of interested variables to select appropriate variables for model building which resembled one of several previous researches [36-38]. We used chi-square [39] to consider correlation between patient information and class label. The results identified that sex and class label were correlated with significant of 0.05 as hypothesis as follows:

$\mathrm{H}_{0}$ : Hepatocellular Carcinoma is independent to sex

$\mathrm{H}_{1}$ : Hepatocellular Carcinoma depends on sex

The results showed that $\mathrm{p}$-value was equal to 0.0465 , which rejected $\mathrm{H}_{0}$. Therefore, Hepatocellular Carcinoma depended on sex.

\section{Data cleansing}

To enhance effectiveness of data analysis, we removed missing values and coped with outliers using a boxplot [39].

\section{Handling imbalanced class}

Relying on the dataset as explained in the data collection section, the dataset of Hepatocellular Carcinoma patients had more than $40 \%$ when compared to normal subject dataset, which may have reduced accuracy. We used Synthetic Minority Oversampling (SMOTE) [40] to solve the problem, which resembled Santos et al. [33]. From our experiment, there were 824 sample subjects, with an equal number of Hepatocellular Carcinoma and normal subjects.

Liver cancer patient detection based on multiple-stage using hybrid classification methods

Once data was preprocessed, as explained in the data preprocessing section, we used this dataset to build a model for liver cancer patient detection and estimating abnormality levels of liver, as described in the next section.

Both models used hybrid classification methods [39,41]. There were 3 classification methods, Multilayer Perceptron (MLP) [39], Logistic Regression [39], and Random Forest [39] by Voting with average of probabilities of each model to select the best answer [39,41], as shown in Figure 2.

MLP [39] is a kind of neural network. Each decision boundary between data is complex, which made it impossible to use only Hyperplane in a single layer to classify data. It was necessary to use a hidden layer to enhance effective classification.

Logistic Regression [39] is a method to analyze the correlation of independent variables. The variable can be either continuous variable or discrete variables. However, detection uses discrete variables by computing probability, as shown in Eq. (1).

$$
P=\frac{1}{1+e^{-y}}
$$

Random Forest [39] is a kind of ensemble method using numerous Decision Trees. This method uses random data and attributes to build a model. Then, the majority vote is used to decide a selected answer. 
http://wjst.wu.ac.th

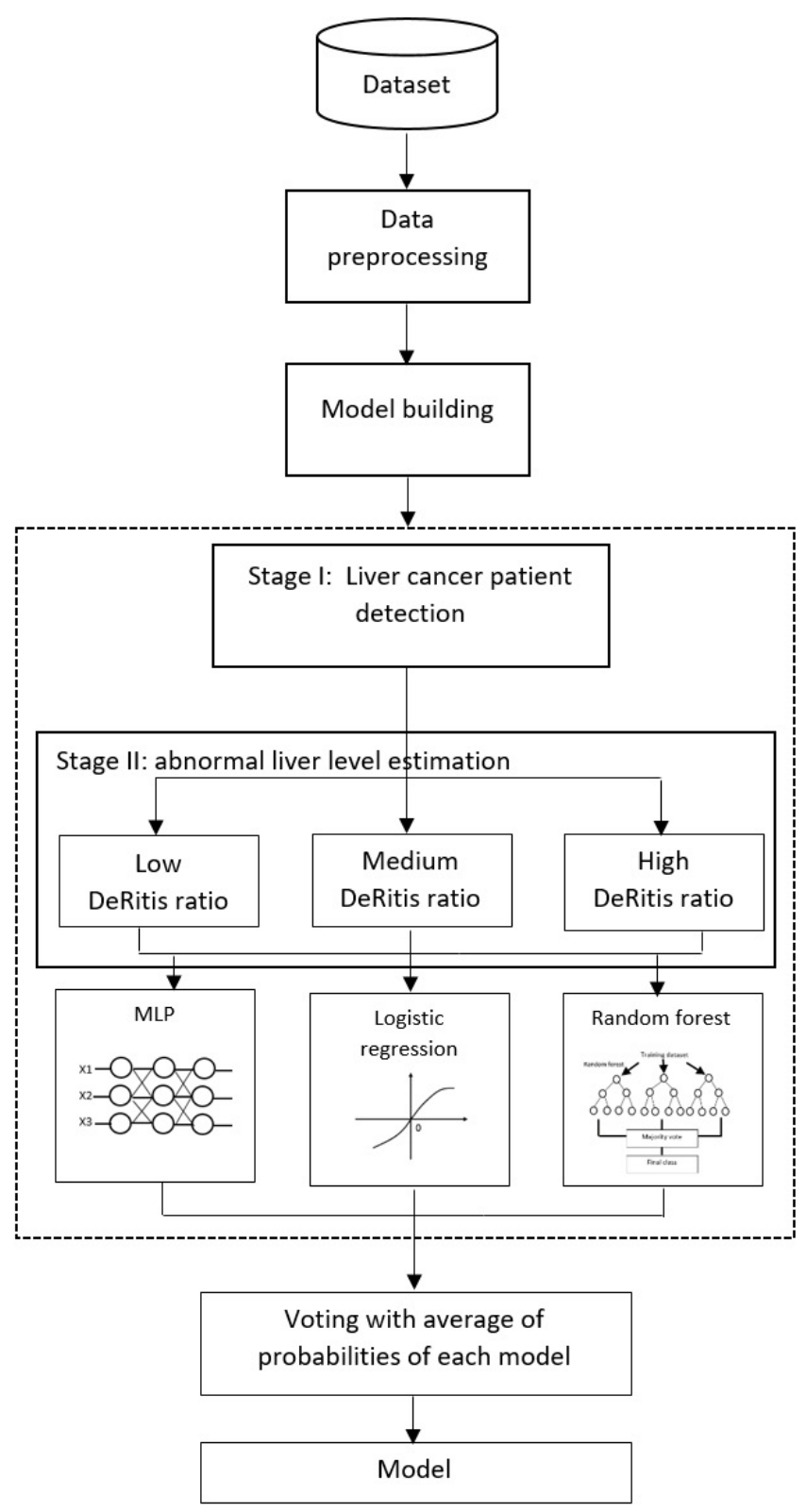

Figure 2 Liver cancer patient detection on multiple-stage using hybrid classification methods.

The parameter determination of the 3 models in stage I is explained as follows:

\section{Stage I: Liver cancer patient detection}

We set up parameters of 3 models as follows: For MLP, there were 3 layers (10 input nodes, 6 hidden nodes, and 2 output nodes). We set learning rate 1.0E-6, momentum 0.7 , batch size 10 , and training time 1,000, as shown in Figure 3. 
http://wjst.wu.ac.th

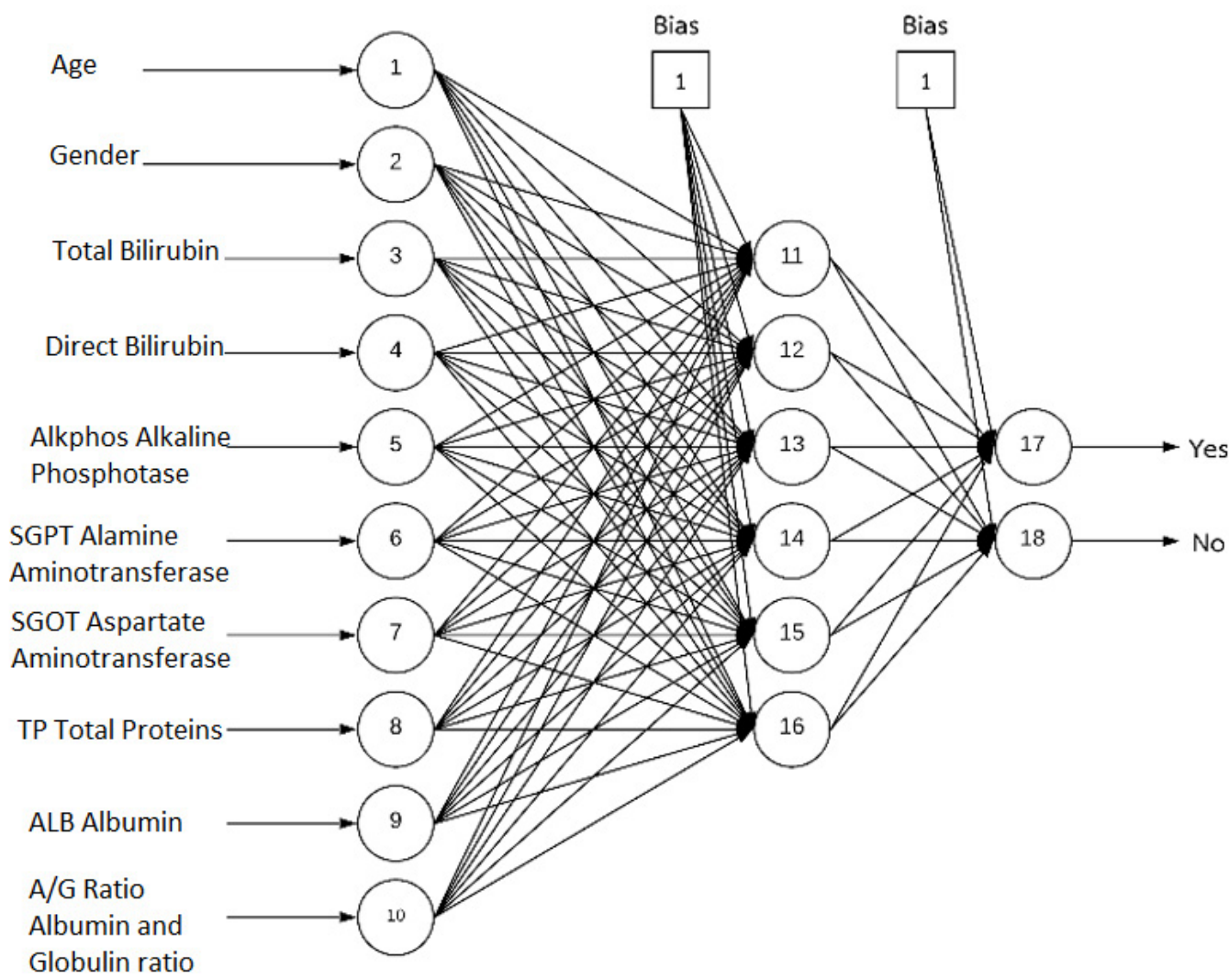

Figure 3 Stage I: Liver cancer patient detection using MLP model.

From Figure 3, "input node consists of Age, Gender, Total Bilirubin, Direct Bilirubin, Alkphos Alkaline Phosphotase, SGPT Alamine Aminotransferase, SGOT Aspartate Aminotransferase, TP Total Proteins, ALB Albumin, A/G ratio, Albumin, and Globulin ratio" [35]. The hidden node was 6 nodes, and output node had 2 answers: YES (Hepatocellular Carcinoma patient) or NO (normal subject).

For Logistic Regression, we set ridge for log-likelihood at 1.0E-8 and batch size equal to 10 .

For Random Forest, we set a number of randomly chosen 10 attributes, number of iterations (100), max depth (20), and batch size (10).

Lastly, hybrid classification methods used a majority vote to select an answer and used an average of probabilities to create a rule of decision.

\section{Stage II: Abnormality level of liver estimation}

Once we obtained the results from the $1^{\text {st }}$ stage, the next stage was abnormal liver level estimation, which used the criterion of the DeRitis Ratio [42] to divide abnormality levels of liver, called ALL (abnormality level of liver) framework. "Partition of abnormality level of liver uses the ratio between aspartate transaminase (AST or SGOT) and alanine transaminase (ALT or SGPT)" [42,43]. The DeRitis Ratio is a part of liver function testing with division criterion [42,43], as seen in Table 2. 
http://wjst.wu.ac.th

Table 2 DeRitis Ratio Decision Limit [42,43].

\begin{tabular}{cccc}
\hline & DeRitis Ratio Decision Limit & \\
\hline Condition & $<\mathbf{1 . 0} \quad \mathbf{1 . 0}$ to $<\mathbf{1 . 5}$ & $\mathbf{1 . 5}$ to $<\mathbf{2 . 0}$ & $>=\mathbf{2 . 0}$ \\
Healthy & Women (up to 1.7) & Children & Neonate \\
Acute Viral Hepatitis & Men (up to 1.3) & Worsening & Fulminant \\
Alcoholic Hepatitis & Resolving & Alcohol Abuse & Acute Hepatitis \\
Chronic Liver Disease & Resolving & Fibrosis risk & Other Causes \\
Muscle Disease & Stable & Resolving & Acute \\
\hline
\end{tabular}

From Table 2, we used Chronic liver disease [44] to divide abnormality levels of liver. The levels were divided into 3: Low, Medium, and High. Level 1 was "Low level". If DeRitis ratio < 1.0, it meant normal, Level 2 was "Medium". If 1.0 to $<2.0$, it meant that it had a risk of the fascia. Level 3 was "High". If DeRitis ratio $>=2.0$, it meant that there were other factors that caused abnormal liver.

The parameter determination of the 3 models in stage II is explained as follows:

For MLP, there were 3 layers (10 input nodes, 6 hidden nodes, and 3 output nodes). We set learning rate at 1.0E-4, momentum (0.3), batch size (20), and training time (500), as shown in Figure 4.

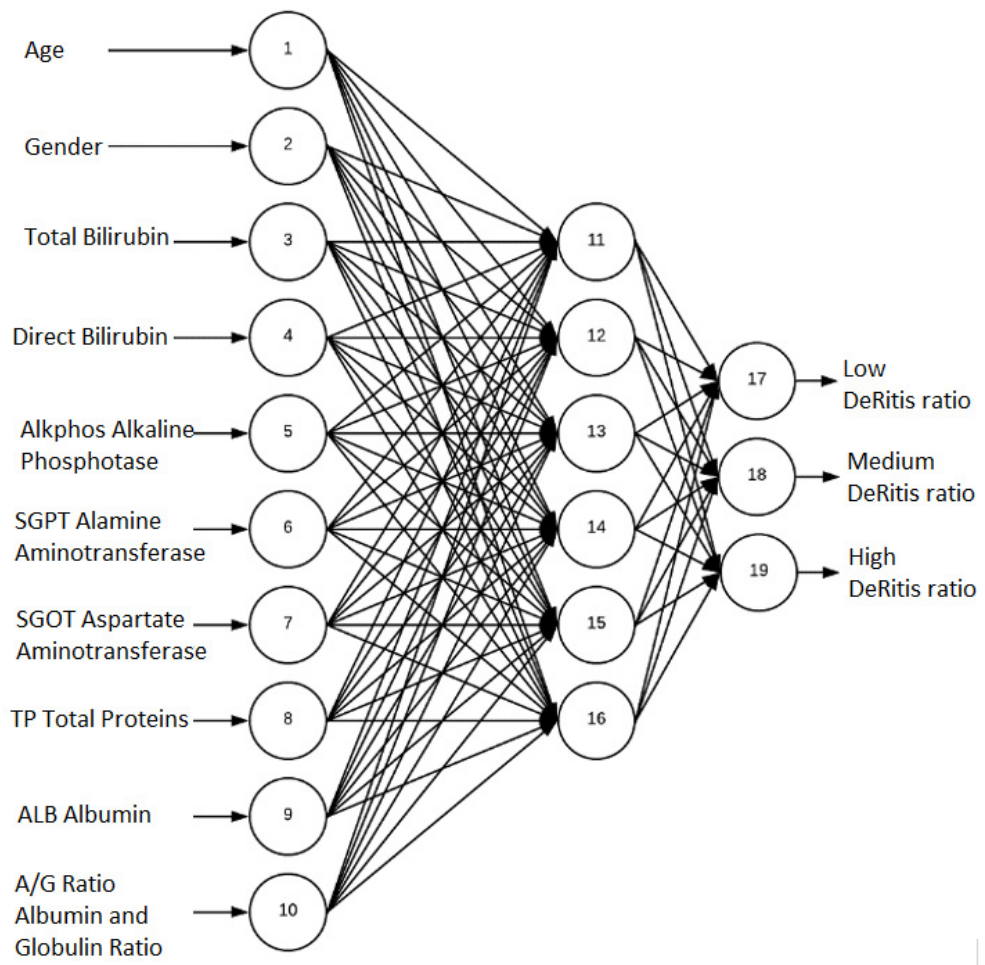

Figure 4 Stage II: Abnormal liver level estimation using MLP model.

From Figure 4, input node consists of 10 attributes as in the model in stage I. The hidden node was 6 nodes, and output node had 3 answers: Low, Medium, or High DeRitis ratio.

For Logistic Regression, we set ridge for log-likelihood at $1.0 \mathrm{E}-8$, and batch size equal to 20 .

For Random Forest, we set number of randomly chosen 10 attributes, number of iterations (100), max depth (10), and batch size (20). 
Moreover, we also used hybrid classification methods, as in the model in stage I.

\section{Experiment result and demonstration}

In this section, we explain the experimental results and case study to demonstrate the effectiveness of our proposed model.

\section{Experiment results and discussions}

In this section, we describe the experimental results with measured effectiveness of liver cancer patient detection and estimating abnormal liver level. There were 824 sample subjects, as explained in the handling imbalanced class section, to build the model and evaluate the effectiveness of the model with train-test split. The dataset was divided, with one part a training dataset with 578 sample subjects (an equal number of Hepatocellular Carcinoma and normal subjects). The rest of the dataset was for testing the model. The results were explained for multiple-stage classification using 3 classifiers. We proposed 3 measurement methods, accuracy, recall, and precision of each classifier, as seen in Table 3.

Table 3 Results of measurement of our proposed model of 3 classifiers.

\begin{tabular}{ccccccc}
\hline & \multicolumn{2}{c}{ MLP } & \multicolumn{2}{c}{ Logistic Regression } & \multicolumn{2}{c}{ Random Forest } \\
\cline { 2 - 6 } & Stage I & Stage II & Stage I & Stage II & Stage I & Stage II \\
Accuracy & $49.19 \%$ & $34.15 \%$ & $70.73 \%$ & $91.46 \% *$ & $76.83 \% *$ & $82.93 \%$ \\
Recall & 0.492 & 0.341 & 0.707 & 0.915 & 0.768 & 0.829 \\
Precision & 0.492 & 0.341 & 0.707 & 0.915 & 0.774 & 0.833 \\
\hline
\end{tabular}

*High accuracy detection

From Table 3, the results showed that Random Forest achieved high accuracy with $76.83 \%$ for liver cancer patient detection (stage I). Therefore, Random Forest was considered the most robust when compared to other classifiers. Second to it in terms of accuracy was Logistic Regression with $70.73 \%$ accuracy. This model also had effectiveness for liver cancer patient detection. Lastly, MLP had the least accuracy of $49.19 \%$.

For abnormality level of liver estimation (stage II), the results showed that Logistic Regression achieved high accuracy of $91.46 \%$. Next, Random Forest had accuracy of $82.93 \%$. In addition, MLP also had the least accuracy of $34.15 \%$.

To enhance performance of detection using individual classifiers, we used hybrid classification methods, as seen in Table 4.

Table 4 Results of measurement of our proposed model of hybrid classifiers.

\begin{tabular}{ccccc}
\hline & \multicolumn{2}{c}{ Our proposed hybrid methods } & Dataset of BUPA Medical Research [45] based on our \\
& & & \multicolumn{2}{c}{ proposed method } \\
\cline { 2 - 5 } & Stage I & Stage II & Stage I & Stage II \\
Accuracy & $78.88 \%$ & $99.83 \%$ & $76.73 \%$ & $98.26 \%$ \\
Recall & 0.789 & 0.998 & 0.767 & 0.983 \\
Precision & 0.803 & 0.998 & 0.767 & 0.983 \\
\hline
\end{tabular}

From Table 4, the results showed that hybrid classification methods for stage I and stage II achieved accuracies of 78.88 and $99.83 \%$, respectively. Therefore, hybrid classification methods outperformed individual classification because it had multiple learning to select the best answer. For example, the system could detect ambiguity of abnormality levels of liver between level 2 and 3 . 
http://wjst.wu.ac.th

In addition, to measure the robustness and non-bias of our proposed model, we used another dataset (BUPA dataset) [45] to estimate the performance of our proposed model. "The dataset consists of 7 attributes such as mcv, alkphos, sgpt, sgot, gammagt, drinks, and selector" [45]. There was a total of 345 records. The dataset split data into a training dataset (200 records) and testing dataset (145 records). The results showed that our proposed model also had effectiveness for liver cancer patient detection and estimating abnormality levels of liver with accuracies of 76.73 and $98.26 \%$, respectively. Therefore, it confirmed that our proposed model could be applied to other datasets. Moreover, the cause of high accuracy was the size of the data. Large volumes of datasets will increase accuracy and enhance the performance of diagnostic support for physicians based on big data approach [46].

\section{Demonstration}

To understand the work process and the benefit of our proposed system, we demonstrate a work simulation of our proposed system. As an example, we use the case of a patient in need of treatment. The patient is a male (age 21 years and with liver data as seen in Figure 5).

\begin{tabular}{|l|c|l|c|}
\hline Age & 21 & Gender & Male \\
\hline Total bilirubin & 0.9 & Direct bilirubin & 0.3 \\
\hline Alkaline phosphotase & 202.0 & Total Proteins & 7.4 \\
\hline Alamine aminotransferse & 22.0 & $\begin{array}{l}\text { Aspartate } \\
\text { aminotransferase }\end{array}$ & 19.0 \\
\hline Ratio AL/GL & 1.2 & Albumin & 4.1 \\
\hline Stage I: HCC Patient Classification & & Liver Patient \\
\hline Stage II : Abnormal Liver Level & & & Low \\
\hline
\end{tabular}

Figure 5 Sample of case study.

From Figure 5, the system uses less than $1 \mathrm{~s}$ processing time after filling in information into the system. The system reports that patient has a probability of liver cancer. Then, the system estimates the abnormality level of the liver at low level, which uses a green icon to display the information for the physician. The results of detection can explain that the patient has the possibility of liver cancer, but the liver is also normal. This information is a benefit to physicians and medical personnel as a support diagnosis. Moreover, to prevent the occurrence of liver cancer, patients may have to assess the risk of other diseases as related by themselves, such as diabetes [47]. 


\section{Conclusions and future work}

This paper proposes multiple stages of liver cancer patient detection and estimating abnormality levels of liver using hybrid classification methods. For our proposed system, we use an Indian liver patient dataset to build a model. The classification methods used to classify multiple stages were MLP, Logistic Regression, and Random Forest. In addition, voting with average of probabilities of each model was used to select an answer for solving a problem. The experimental results showed that Random Forest had $76.83 \%$ accuracy for liver cancer patient detection (stage I). Logistic Regression achieved high accuracy with $91.46 \%$ for abnormality levels of liver estimation (stage II).

Moreover, a hybrid classification method was used to detect liver cancer patients in stage I and estimating abnormality levels of liver in stage II. The results showed that our proposed model could detect stage I and stage II with accuracies of 78.88 and $99.83 \%$, respectively.

Furthermore, we tested our proposed model with another dataset [45] to enhance effectiveness and reduce nonbiased. The results showed that accuracy of detection in stage I and stage II was 76.73 and $98.26 \%$, respectively. Therefore, this confirmed that our proposed system was robust and could be applied to other models.

For future work, we plan to develop an intelligent system to support the decision making of physicians for diagnosis and treatment. The system will integrate liver cancer patient detection and estimating abnormality levels of liver to use in real situations.

\section{References}

[1] M Pasha and M Fatima. Comparative analysis of meta learning algorithms for liver disease detection. J. Softw. 2017; 12, 923-33.

[2] H Pakhale and DK Xaxa. A survey on diagnosis of liver disease classification. Int. J. Eng. Tech. 2016; 2, 132-8.

[3] EM Hashem and MS Mabrouk. A study of support vector machine algorithm for liver disease diagnosis. Am. J. Intell. Syst. 2014; 2, 9-14.

[4] S Sontakke, J Lohokare and R Dani. Diagnosis of liver diseases using machine learning. In: Proceedings of the Emerging Trends and Innovation in ICT, India, 2017, p. 129-33.

[5] Office of Policy and Strategy of the Ministry of Public Health. Public health statistics A.D. 2008. WVO office of Printing Mill, Bangkok, Thailand.

[6] JD Yang and LR Roberts. Hepatocellular carcinoma: A global view. Nat. Rev. Gastroenterol. Hepatol. 2010; 7, 448-58.

[7] T Kitiyakara. Advances in biomarkers for HCC. Thai J. Hepatol. 2018; 1, 29-32.

[8] T Higashi, SL Friedman, Y Hoshida. Hepatic stellate cells as key target in liver fibrosis. Adv. Drug Deliv. Rev. 2017; 121, 27-42.

[9] JT Coon, G Rogers, P Hewson, D Wright, R Anderson, M Cramp, S Jackson, S Ryder, A Price and K Stein. Surveillance of cirrhosis for hepatocellular carcinoma: Systematic review and economic analysis. Health Technol. Assess. 2007; 11, 1-206.

[10] SL Chan, F Mo, PJ Johnson, DYW Siu, MHM Chan, WY Lau, PBS Lai, CWK Lam, W Yeo and $\mathrm{SCH} \mathrm{Yu}$. Performance of serum $\alpha$-fetoprotein levels in the diagnosis of hepatocellular carcinoma in patients with a hepatic mass. Hepato Pancreato Biliary (HPB) 2013; 16, 366-72.

[11] H Kaur, S Bhalla and GPS Raghava. Classification of early and late-stage liver hepatocellular carcinoma patients from their genomics and epigenomics profiles. PLoS One 2019; 14, 1-27.

[12] R Panthong and A Srivihok. Liver cancer classification model using hybrid feature selection based on class-dependent technique for the central region of Thailand. Information 2019; 10, 1-20.

[13] S Vijayarani and S Dhayanand. Liver disease prediction using SVM and Naïve Bayes algorithms. Int. J. Sci. Eng. Tech. Res. 2015; 4, 816-20.

[14] M Rouhani and MH Haghighi. The diagnosis of hepatitis diseases by support vector machine and artificial neural networks. In: Proceedings of Association of Computer Science and Information Technology, Singapore, 2009, p. 456-58. 
[15] A Alaybeyoglu and N Mulayim. A design of hybrid expert system for diagnosis of breast cancer and liver disorder. In: Proceedings of Research in Education and Science, Turkey, 2018, p. 345-53.

[16] J Jacob, JC Mathew, J Mathew, E Issac. Diagnosis of liver disease using machine learning techniques. Int. J. Eng. Technol. 2018; 5, 4011-14.

[17] P Kumar and RS Thakur. Liver disorder detection using variable- neighbor weighted fuzzy K nearest neighbor approach. Multimed. Tools Appl. 2021. https://doi.org/10.1007/s11042-019-079783.

[18] TR Baitharul and S K Pani. Analysis of data mining techniques for healthcare decision support system using liver disorder dataset. In: Proceedings of Computational Modeling and Security, India, 2016, p. 862-70.

[19] MKS Maaitah, R Abiyev and IJ Bush. Intelligent classification of liver disorder using fuzzy neural system. Int. J. Adv. Comput. Sci. Appl. 2017; 8, 25-31.

[20] A Kumar and N Sahu. Categorization of liver disease using classification techniques. Int. J. Res. Appl. Sci. Eng. Technol. 2017; 5, 826-8.

[21] M Aiswarya, S Srinivas and AGH Narayanan. Illustration of random forest and naïve bayes algorithms on Indian liver patient dataset. Int. J. Pure. Appl. Math. 2018; 119, 585-95.

[22] MB Priya, PL Juliet and PR Tamilselvi. Performance analysis of liver disease prediction using machine learning algorithms. Int. J. Eng. Technol. 2018; 5, 206-11.

[23] A Pathan, D Mhaske, S Jadhav, R Bhondave and K Rajeswari. Comparative study of different classification algorithms on ILPD dataset to predict liver disorder. Int. J. Appl. Sci. Eng. Technol. $2018 ; \mathbf{4 5}, 388-94$.

[24] S Muthuselvan, S Rajapraksh, K Somasundaram and K Karthik. Classification of liver patient dataset using machine learning algorithms. Int. J. Eng. Technol. 2018; 7, 323-26.

[25] G Rajesh and SMA Priyadharson. Liver cancer detection and classification based on optimum hierarchical feature fusion with PeSOA and PNN classifier. Biomed. Res. 2018; 29, 22-32.

[26] A Das, P Das, SS Panda and S Sabut. Detection of liver cancer using modified fuzzy clustering and decision tree classifier in CT images. Math. Meth. Pattern Recognit. 2019; 29, 201-11.

[27] M Chen, B Zhang, W Topatana, J Cao, H Zhu, S Juengpanich, Q Mao, H Yu and X Cai. Classification and mutation prediction based on histopathology H\&E images in liver cancer using deep learning. NPJ Precis. Oncol. 2020; 4, 1-7.

[28] S Almotairi, G Kareem, M Aouf, B Almutairi and MAM Salem. Liver tumor segmentation in CT scans using modified SegNet. Sensors 2020; 20, 1-13.

[29] N Nahar, F Ara and MA Neloy. A comparative analysis of the ensemble method for liver disease prediction. In: Proceedings of International Conference on Innovation in Engineering and Technology, Bangladesh, 2019, p. 1-6.

[30] S Sathurthi and K Saruladha. Prediction of liver cancer using random forest ensemble. Int. J. Pure Appl. Math. 2017; 116, 267-73.

[31] C Aswathy, Code Project. Liver patient dataset classification using the intel distribution for Python, Available at: https://software.intel.com/en-us/articles/liver-patient-dataset-classification-using-theintel-distribution-for-python, accessed June 2019.

[32] GI Rajathi and GW Jiji. Chronic liver disease classification using hybrid whale optimization with simulated annealing and ensemble classifier. Symmetry 2019; 11, 1-21.

[33] MS Santos, PH Abreu, PJ Garcia-Laencina, A Simao and A Carvalho. A new cluster-based oversampling method for improving survival prediction of hepatocellular carcinoma patients. $J$. Biomed. Inform. 2015; 58, 49-59.

[34] A Singh and B Pandey. An euclidean distance based KNN computational method for assessing degree of liver damage. In: Proceedings of Inventive Computation Technologies, India, 2016, p. 14.

[35] ILPD (Indian Liver Patient Dataset). Data Set, UCI Machine Learning Repository, Available at: https://archive.ics.uci.edu/ml/datasets/ILPD+ (Indian+Liver+Patient+Dataset), accessed April 2019.

[36] K Swapna and P Babu. Critical analysis of Indian liver patients dataset using ANOVA method. Int. J. Eng. Technol. 2017; 7, 19-33. 
http://wjst.wu.ac.th

[37] SH Adil, M Ebrahim, K Raza, SSA Ali and MA Hashmani. Liver patient classification using logistic regression. In: Proceedings of $4^{\text {th }}$ Computer and Information Sciences, Malaysia, 2018, p. $1-5$.

[38] BV Ramana, SP Babu and NB Venkateswarlu. A critical comparative study of liver patients from USA and India: An exploratory analysis. Int. J. Comput. Sci. 2012; 9, 506-16.

[39] J Han and M Kamber. Data mining concepts and techniques. Morgan Kaufmann Publishers, 2011.

[40] NV Chawla, KW Bowyer, LO Hall and WP Kegelmeyer. SMOTE: Synthetic minority oversampling technique. J. Artif. Intell. Res. 2002; 16, 321-57.

[41] G Tuysuzoglu, D Birant and A Pala. Ensemble methods in environmental data mining. IntechOpen, 2018, p. 1-16.

[42] M Botros and KA Sikaris. The deritis ratio: The test of time. Clin. Biochem. 2013; 34, 117-30.

[43] JR Mera, B Dickson and M Feldman. Influence of gender on the ratio of serum aspartate aminotransferase (AST) to alanine aminotransferase (ALT) in patients with and without hyperbilirubinemia. Dig. Dis. Sci. 2008; 53, 799-802.

[44] Chronic Liver Disease. Available at: https://stanfordhealthcare.org/medical-conditions/liverkidneys-and-urinary-system/chronic-liverdisease.html ${ }_{2}$ accessed June 2019.

[45] BUPA Medical Research. UCI Machine Learning Repository, Available at: https://archive.ics.uci.edu/ml/datasets/liver+disorders, accessed April 2019.

[46] S Mathivanan and P Jayagopal. A big data virtualization role in agriculture: A comprehensive review. Walailak J. Sci. Tech. 2019; 16, 55-77.

[47] P Jinpon, M Jaroensutasinee and K Jaroensutasinee. Risk assessment of type 2 diabetes mellitus in the population of chonburi, Thailand. Walailak J. Sci. Tech. 2017; 14, 25-33. 